\title{
Editorial: ASE 2011 conference trip report
}

\author{
Robert J. Hall
}

Received: 21 November 2011 / Accepted: 23 November 2011 / Published online: 7 December 2011

(C) Springer Science+Business Media, LLC 2011

The 2012 volume of $A S E J$ promises to be both strong and diverse, reflecting the wide scope of our field. This issue starts us off well with a representative sample of interesting research contributions.

- Multi-agent programs model beliefs, desires, and intentions to collaboratively achieve complex goals. In "Model checking agent programming languages", Dennis, Fisher, Webster, and Bordini describe the first comprehensive verification system for model checking multi-agent programs. Their system, based on an abstraction layer that maps agent semantics down to the underlying model checker, is extensible and can accommodate heterogeneous agent formalisms.

- In "Prioritising test cases with string distances", Ledru, Petrenko, Boroday, and Mandran discover that test case prioritization can be done with access to neither the source code under test nor a specification. Instead, they calculate a string distance measure between texts of test cases and define a prioritization algorithm that uses it. They explore different distance metrics and show that, using a Manhattan distance metric, their technique significantly outperforms random permutation.

- In "Refactoring techniques for aggressive object inlining in java applications", Asher, Gal, Haber, and Zalmanovici focus on making the object inlining (OI) technique practical and useful. Object inlining replaces an object having a field that references a second object with a single object that contains the fields of the second object directly in itself. They describe an IDE-integrated, semi-automated technique that first suggests parts of the code that can be refactored to enable OI, and then, in a second phase, carries out identified OI operations prior to normal compilation. They show in a case study the promise of the approach.

R.J. Hall (殴

AT\&T Labs Research, Florham Park, NJ 07932, USA

e-mail: Bob.ASEJ@gmail.com 


\section{The 26th international conference on automated software engineering}

ASE 2011 was held during the week of 7 November at the brand new Oread Hotel in Lawrence, Kansas, USA. Adjacent to the University of Kansas, the hotel from the outside looks like a hybrid between a factory (four tall smokestacks on top) and a castle (terraced stone). Inside, the motif is all castle, having corridors lined with stone and laid out to suggest a castle interior. The weather started out rainy and cold early, but turned nice for the three days of the main conference.

General Chair Perry Alexander of KU and his team did a great job putting on a smoothly running and interesting conference. Of special note was the banquet, which featured excellent Kansas barbecue cuisine and after-dinner entertainment by a bluegrass musical group. A new feature of this year's conference program was that authors of demonstrations and short papers were given 7-minute speaking slots in addition to a poster/demo session. This gave attendees two opportunities to experience the content in their choice of format. In particular, one could listen to the talks in order to prepare for and focus on particular demos and short papers to probe more deeply the next day at the interactive session, as opposed to the traditional wandering around, trying to read all the posters and then ask questions. The demos and short papers continue to be among the liveliest and most interesting sessions of the conference, and this year's addition of preview talks enhanced that even further.

In the conference program, several ideas and themes struck me as especially interesting.

- Matt Dwyer, in his keynote, laid out a vision for verification tools working together to achieve better results faster than single tools alone. He visualized this as a matrix of correctness properties versus behaviors, with tools somehow being engineered to describe to each other the portions of this 2-D space each covers so that other tools can abstract portions and simplify their own reasoning. He gave an example that could reduce the ambiguity of a program analysis by using a different tool to show that several converging control flows in the middle of a function all result in the same constrained set of possibilities. This result is then propagated by the program analyzer, instead of the overly conservative value sets it had itself inferred at the point in question. Of course, this grand vision of a patchwork of tools covering the matrix by working together is far from reality, as no one has as yet made significant progress on this tool interoperation problem.

- Tim Menzies and his coauthors gave an argument that previous work on data mining universal laws or relationships governing decision support tools like defect predictors or cost estimators is on the wrong track, because of the instability problem. That is, while one case study concludes one such relationship, it will generally not be replicated in studies on data from other projects. The authors propose that, rather than looking for ever better inference algorithms, the community should instead focus on searching for islands of regularity within the data space. Essentially, one finds a body of data similar to that obtained from one's own shop and then uses regularities inferred from the similar data. This seems to me to be a fairly radical and mildly surprising shift in the concept of this field.

Echoing this theme of instability in empirical software engineering results, the paper by Posnett et al., discusses instability of results caused by looking at the 
wrong level of hierarchy in system descriptions (i.e. files vs packages vs modules) and suggests this issue needs more study.

- Automated empirical techniques, those in which interesting information is sought in large poorly structured spaces or knowledge sources, are a popular topic among the long papers this year. Knowledge sources include natural language text, such as bug reports and online discussion forums, process data relating to cost, schedule, and defects, static information like code structure, and dynamic execution data gathered during program runs. Counting data and information mining, machine learning, and knowledge acquisition, I count at least 8 long papers out of 37 in this area, including one of the distinguished paper award papers. There was also a post-conference workshop (MALETS) on machine learning technologies in software engineering. This matches a recent surge in interest in this topic area among Journal submissions as well, and reflects intellectual trends toward information mining one sees elsewhere these days in the computer science literature.

- Several of us participated in the first-ever ASE outdoor multiplayer smartphone game play session I organized. For the historical record, Sunny Wong won the iTESS game, a hide-and-seek "tag" style game with virtual weapons and surveillance drones. John Grundy won the iTron game, which involves avoiding crashing into virtual trails left by players as they move around in the real world. While fun in themselves, the connection to software engineering was made by capturing wireless data during the games, and then taking it inside and using my Capture Calculus Toolset on the data during the demo session to demonstrate requirements engineering and V\&V of distributed embedded systems (DESs). DESs in general, and mobile applications in particular, are, of course, hot topics in the industry. Other DES examples include smart homes, smart grids, and other ubiquitous computing scenarios. DESs are rife with new software engineering research problems as well as novel spins on old problems, and I expect to see increasing attention focused on DES software engineering research problems.

Finally, in their annual meeting, the ASE Steering Committee decided, among other things, to hold ASE 2014 in Västerås, Sweden, during September 2014, with Ivica Crnkovic of Mälardalen University as General Chair. Recall that ASE will be in Essen, Germany (Michael Goedicke, General Chair) in September 2012, and it will be in Palo Alto, California, USA (Ewen Denney, General Chair) in November 2013.

Please remember these are only my own subjective experiences and opinions, no doubt biased by the fraction of the conference activities I was able to attend. That said, I'd be happy to receive your thoughts and reactions to this editorial; feel free to email me at Bob.ASEJ@gmail.com. 